\title{
Variation of Saturn's UV aurora with SKR phase
}

\author{
J. D. Nichols, ${ }^{1}$ B. Cecconi, ${ }^{2}$ J. T. Clarke, ${ }^{3}$ S. W. H. Cowley, ${ }^{1}$ J.-C. Gérard, ${ }^{4}$ A. Grocott, ${ }^{1}$ \\ D. Grodent, ${ }^{4}$ L. Lamy, ${ }^{5}$ and P. Zarka ${ }^{2}$
}

Received 25 May 2010; revised 16 June 2010; accepted 21 June 2010; published 6 August 2010.

[1] It is well known that a wide range of kronian magnetospheric phenomena, including the Saturn kilometric radiation (SKR), exhibit oscillations near the planetary rotation period. However, although the SKR is believed to be generated by unstable auroral electrons, no connection has been established to date between diurnal SKR modulations and UV auroral power. We use an empirical SKR phase determined from Cassini observations to order the 'quiet time' total emitted UV auroral power as observed by the Hubble Space Telescope in programs during the interval 20052009. Our results indicate that both the northern and southern UV powers are dependent on SKR phase, varying diurnally by factors of $\sim 3$. We also show that the UV variation originates principally from the morning half of the oval, consistent with previous observations of the SKR sources. Citation: Nichols, J. D., B. Cecconi, J. T. Clarke, S. W. H. Cowley, J.-C. Gérard, A. Grocott, D. Grodent, L. Lamy, and P. Zarka (2010), Variation of Saturn's UV aurora with SKR phase, Geophys. Res. Lett., 37, L15102, doi:10.1029/2010GL044057.

\section{Introduction}

[2] A key property of the Saturn kilometric radiation (SKR) is that its intensity pulses at varying periods that are near to that of planetary rotation [e.g., Kurth et al., 2008; Gurnett et al., 2009], and a major discovery of the Cassini mission has been the surprising ubiquity of other oscillatory magnetospheric phenomena with similar periods [e.g., Cowley et al., 2006; Carbary et al., 2008; Nichols et al., 2008]. Kurth et al. [2005] demonstrated a correlation between UV auroral power and solar wind shock-induced enhancements in SKR power, and Mitchell et al. [2009] presented a few case studies of brightenings of the dawnside UV aurora observed in both HST and Cassini Ultraviolet Imaging Spectrometer (UVIS) data, that they associated with recurrent energisation of plasma and SKR intensifications. However, no periodic variations in the auroral power at the SKR period have been reported, despite the fact that the SKR radio sources map along the magnetic field to the auroral oval [Lamy et al., 2009]

\footnotetext{
${ }^{1}$ Department of Physics and Astronomy, University of Leicester, Leicester, UK.

${ }^{2}$ LESIA, Observatoire de Paris, Université Paris Diderot, CNRS, Meudon, France.

${ }^{3}$ Center for Space Physics, Boston University, Boston, Massachusetts, USA.

${ }^{4}$ Laboratoire de Physique Atmosphérique et Planétaire, Université de Liège, Liège, Belgium.

${ }^{5}$ Space and Atmospheric Physics Group, Imperial College London, London, UK.

Copyright 2010 by the American Geophysical Union. 0094-8276/10/2010GL044057
}

and the candidate mechanisms for SKR generation invoke unstable auroral electron distributions [e.g., $W u$ and Lee, 1979]. This apparent disconnect may simply be due to the relative paucity of auroral images obtained by the Hubble Space Telescope (HST), which are generally limited to a few days of observations in any one year. However, since Cassini's arrival at Saturn, a number of HST programs have now built up a substantial archive of auroral images obtained with high-sensitivity cameras such as the Advanced Camera for Survey (ACS) onboard HST. In this paper we order UV auroral power values computed from images obtained over 2005-2009 by the phase of the SKR intensity oscillations determined empirically from observations made by the Cassini Radio and Plasma Wave Science (RPWS) instrument [Gurnett et al., 2004] in order to determine whether a relation between the two phenomena exists.

\section{Data}

[3] We first discuss the UV auroral power values. We employ ACS images obtained during HST programs which executed in Oct 2005, Jan 2007, Feb 2008, and Jan-Mar 2009. The methods of reduction and extraction of the total emitted power values from these images have been extensively discussed previously [see, e.g., Gérard et al., 2006; Clarke et al., 2009; Nichols et al., 2009], such that here we simply note that the total power is computed by summing the auroral emission over the whole auroral region in the images, while the powers emitted from the dawn (AM) and dusk (PM) sides are obtained by summing over each half of the region. We separate these two halves because the SKR is thought to originate principally from the morning [Galopeau et al., 1995; Lamy et al., 2009], and the UV auroras are generally brighter on the dawnside than the duskside [Grodent et al., 2005]. Southern power values are available for all years, while the first time the northern auroras became visible was during the 2009 equinoctial program [Nichols et al., 2009]. Saturn's auroral emission brightens and expands significantly in response to interplanetary shocks [Prangé et al., 2004; Clarke et al., 2009], an effect which occurs independently of SKR phase and swamps all other variations in the auroral morphology. We thus only consider images in which the auroras exhibit the 'quiet time' oval morphology of the kind observed in Oct 2005 [Gérard et al., 2006]. Our data set then consists of 209 , or $\sim 77 \%$, of the total of 273 images available.

[4] The UV powers are ordered using an empirical determination of the phase of the SKR oscillation $\phi_{S K R}$, defined separately for both the north and south such that the SKR intensity is statistically maximum at $0^{\circ}$. We also employ SKR powers $P_{\text {SKR }}$ in $\mathrm{dB}$ above $1 \mathrm{~W} \mathrm{sr}^{-1}$, integrated over 40 $1000 \mathrm{kHz}$ [Lamy et al., 2008], and high-pass filtered at $5 \mathrm{~h}$ in 

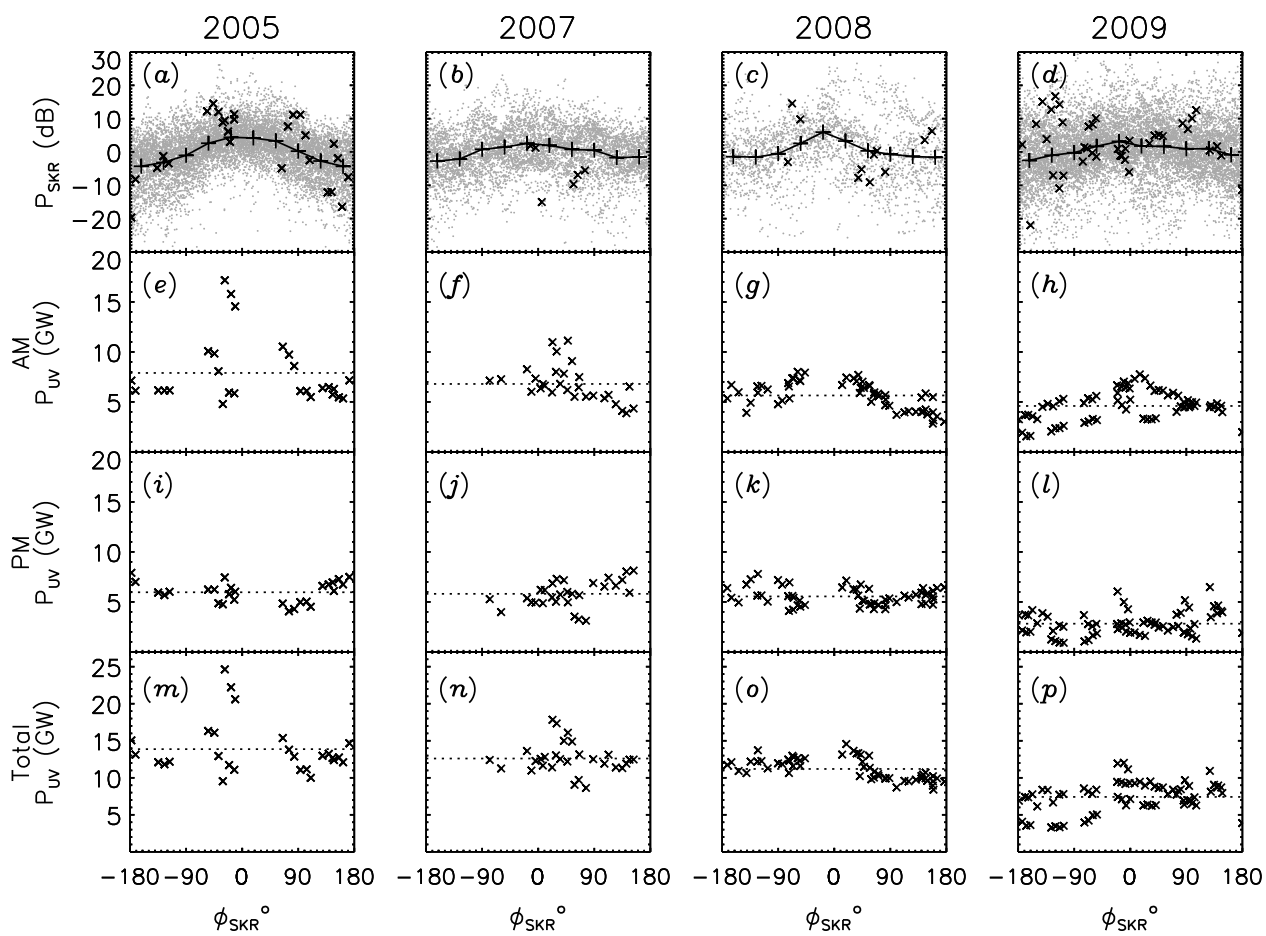

Figure 1. Plot showing the (a-d) filtered southern SKR power $P_{\text {SKR }}$, and $(\mathrm{e}-\mathrm{h})$ dawnside, (i-l) duskside, and $(\mathrm{m}-\mathrm{p})$ total southern UV power values $P_{U V}$ versus empirical SKR phase $\phi_{\text {SKR }}$ for years 2005 (column 1), 2007 (column 2), 2008 (column 3) and 2009 (column 4). In Figures 1a-1d the joined pluses show the mean SKR powers in ten $36^{\circ}$-wide phase bins and the crosses show the values at the times of the HST images. The horizontal dotted lines show the mean UV power for each plot.

order to reduce low order variations due to e.g. the motion of Cassini. For further details see the auxiliary material. ${ }^{1}$

\section{Analysis}

[5] We first show in Figures 1a-1d the southern SKR power over $\sim 30$ day intervals encompassing each set of HST observations versus southern SKR phase $\phi_{\text {SKR. Also shown }}$ by the crosses in Figures $1 \mathrm{a}-1 \mathrm{~d}$ are the southern SKR powers where available corresponding to the specific times the HST images were obtained (corrected for light travel time between Saturn and the Earth) and the joined pluses show the means of all the data in ten $36^{\circ}$ bins. We note from Figures 1a-1d that in these folded data and at HST sampling intervals, the SKR pulsing is not always obvious, although it is clearly present in the unfolded data shown in Figure S1 in the auxiliary material. Figure 1 also shows the dawnside (Figures 1e-1h), duskside (Figures 1i-1l) and total (Figures $1 \mathrm{~m}-1 \mathrm{p}$ ) southern UV auroral powers $P_{U V}$, versus southern SKR phase $\phi_{\text {SKR }}$ separately for each year. Although significant scatter is present in the UV powers, all panels in the dawnside row (Figures 1e-1h) exhibit a trend for higher power values to occur toward $0^{\circ}$ phase, i.e., near where the SKR peaks. On the other hand, the power values in the duskside row (Figures 1i-11) do not exhibit an obvious dependence on SKR phase. The total powers shown in Figures $1 \mathrm{~m}-1 \mathrm{p}$, which are the sum of the two previous rows, thus also exhibit the trend for higher powers to occur

\footnotetext{
${ }^{1}$ Auxiliary materials are available in the HTML. doi:10.1029/ 2010GL044057.
}

toward the middle of the plot, although this is less clear in this case since these also contain the scatter imparted by the duskside values. The ratios between the maximum and minimum UV power values in Figures $1 \mathrm{e}-1 \mathrm{p}$ are all in the range $\sim 2-7$, with a mean ratio of $\sim 3.2$.

[6] In order to consolidate these results, we show these data superposed in Figure 2. First, Figure 2a shows the southern SKR powers at the specific times of the HST images shown in Figures 1a-1d, and also shown by the joined crosses are the mean powers in ten $36^{\circ}$ bins. This panel reinforces the point that the SKR pulsing is not greatly apparent at HST sampling intervals. Figures $2 b-2 d$ show the superposed dawnside, duskside and total southern UV powers, respectively, and we note that here the powers are shown as deviations from each year's mean indicated by the horizontal lines in Figure 1, since there are systematic differences between the powers derived each year caused by, e.g., Saturn's seasonal progression. Table 1 also shows the statistics of the variation of the UV and SKR powers with SKR phase, which are, from top to bottom: the peak-to-peak amplitudes $\delta$ of the solid lines in Figure 2; the mean standard errors between the individual powers in each bin and the bin means $\bar{\sigma}$, indicating the spread around the solid lines; the linear correlation coefficients $r$ between $\Delta P_{U V}$ and $\cos \phi_{\mathrm{SKR}}$, which provides a zeroth-order estimation of the relation between $\Delta P_{U V}$ and $\phi_{\mathrm{SKR}}$; and the corresponding false-alarm probabilities $p$ for these correlations, i.e., the probability that $\Delta P_{U V}$ and $\cos \phi_{\mathrm{SKR}}$ are actually uncorrelated, given by $p=\operatorname{erfc}(|r| \sqrt{N / 2})$ where $N$ is the number of data points [Press et al., 2007]. The SKR results are derived from the SKR powers at the specific times of the HST images, in order 
South 2005, 2007, 2008, \& 2009

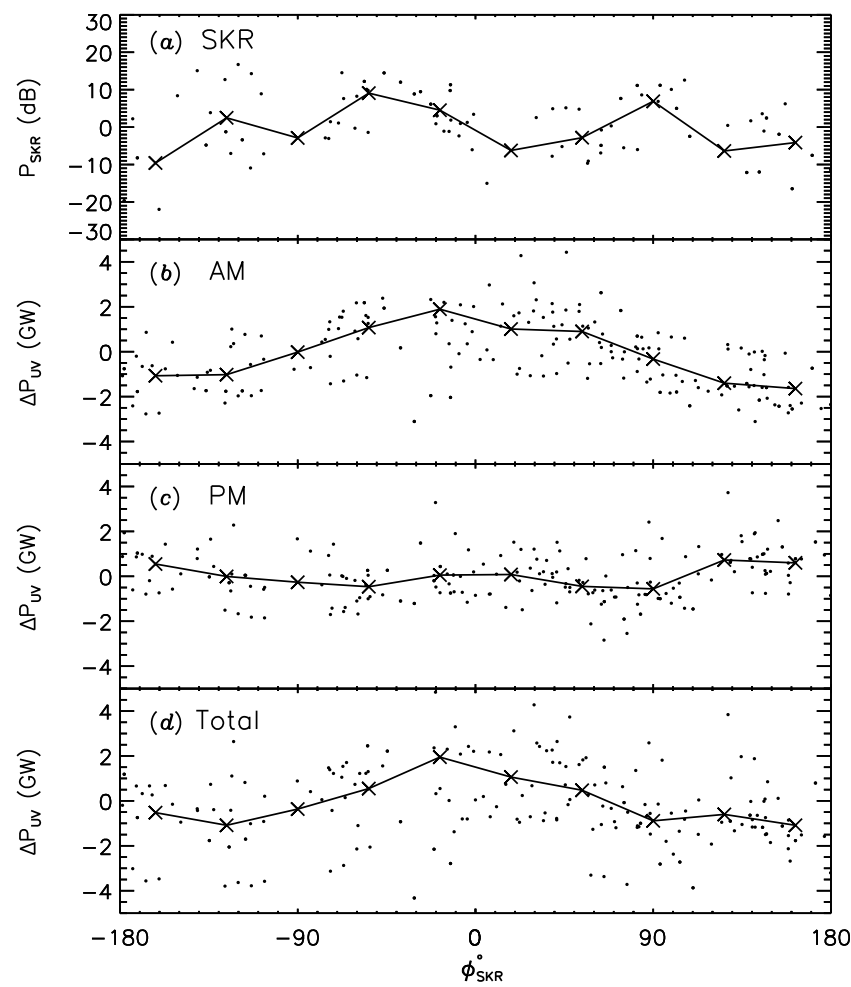

Figure 2. Plot showing the superposed epoch results. (a) Filtered logarithm of the southern SKR powers $P_{\mathrm{SKR}}$, and the deviations of the southern UV power values from each year's mean $\Delta P_{U V}$ for (b) the dawnside, (c) the duskside, and (d) in total. Crosses connected by the solid lines show the mean values in ten $36^{\circ}$-wide phase bins.

to compare with the HST results. The trend for generally increased power values near to $0^{\circ}$ is most clearly apparent in the dawnside data shown in Figure 2b. It is also reflected in the statistics in column 1 of Table 1, in which the spread around the solid line in Figure $2 b$ is significantly less than its peak-to-peak amplitude, and the correlation coefficient of $\sim 0.6$ between $\Delta P_{U V}$ and $\cos \phi_{\mathrm{SKR}}$ is highly significant. In contrast, the duskside data shown in Figure $2 \mathrm{c}$ whose statistics are shown in column 2 of Table 1 exhibit a similar degree of scatter and possibly a slight anti-correlation with $\cos \phi_{\mathrm{SKR}}$, as may be expected for a rotating field-aligned current system, but this result is weakly supported by these data. The total UV power values shown in Figure $2 \mathrm{~d}$ whose statistics are shown in column 3 of Table 1, represent the expected combination of the above results in that the dawnside dependence on $\phi_{\mathrm{SKR}}$ is present but less clear due to the increased scatter imparted by the duskside emission. The statistics of the variation of the SKR sampled at the cadence of HST shown in column 4 of Table 1 are not significantly different to the UV results.

[7] An alternative analysis of the dependence of Saturn's southern UV auroral brightness on $\phi_{\text {SKR }}$ and local time (LT) is shown in Figure 3. Each image was averaged over $0.5 \mathrm{~h}$ LT bins, and for each bin the maximum auroral brightness between $7^{\circ}$ and $22^{\circ}$ colatitude (a range which encompasses the auroral oval in all images used in this study) was obtained. We note that the maximum brightness represents a related but subtly different parameter to the total power previously plotted, but we use this since here the projections to a latitudelongitude grid do not conserve energy, while the intensities remain unchanged. The maximum intensity values thus obtained were then averaged over $10^{\circ} \phi_{\text {SKR }}$ bins, and the results are shown in Figure 3a, in which the maximum brightness is shown in LT-SKR phase space. The LT range is limited to the dayside region since this was observable in all years, and avoids the region very close to the planet's limb where intensities may be significantly affected by limb-brightening. The same trend on the dawnside (i.e., the left half of Figure 3a) as shown previously is apparent, i.e., brighter auroras occur toward $0^{\circ}$ SKR phase, while low intensities occur near to $\pm 180^{\circ}$. Interestingly, although not robustly present in the previous plots of auroral power, it is possible that the opposite trend, i.e., lower intensities toward $0^{\circ}$ phase, is apparent on the duskside, although the variability is much less than for the dawnside, such that the total power is dominated by the dawnside variation. Such variation would be expected of isolated arcs of emission such as those observed by Grodent et al. [2005], rotating at the SKR period. Figures $3 \mathrm{~b}$ and $3 \mathrm{c}$ show representative images $\sim 180^{\circ}$ apart in SKR phase. From these images it is clear that the variation with $\phi_{\text {SKR }}$ is such that the dawnside auroral oval is brighter near $0^{\circ}$, where the SKR intensity peaks, than near $\pm 180^{\circ}$, where the SKR and dawnside auroral emission are both dim.

[8] The analysis discussed hitherto has considered solely the southern auroral emission with respect to the phase of the southern SKR emission, and we finally consider the northern auroral emission imaged in 2009. The results are shown in Figure 4 in a similar format to Figure 1, except that in Figure 4a the means of all the data in ten bins are shown by pluses joined by dashed lines and the joined crosses show the bin means of SKR at HST times, in Figures $4 b-4 d$ the joined crosses show the bin means of UV powers, and the powers are plotted versus northern SKR phase. There are fewer data points for the north than for the south, but similar behaviour to the southern emission is apparent, arguably exhibited more robustly, in that for the dawnside (and total) emission, elevated emission occurs toward $0^{\circ}$ phase, while weaker anti-phase behaviour occurs on the duskside. These

Table 1. Statistics of Variation of UV Power With SKR Phase ${ }^{a}$

\begin{tabular}{|c|c|c|c|c|c|c|c|c|}
\hline & \multicolumn{4}{|c|}{ South } & \multicolumn{4}{|c|}{ North } \\
\hline & Dawnside & Duskside & Total & SKR & Dawnside & Duskside & Total & SKR \\
\hline$\delta$ & 3.53 & 1.28 & 3.11 & 18.7 & 4.88 & 1.43 & 4.11 & 35.1 \\
\hline $\bar{\sigma}$ & 1.67 & 0.98 & 2.12 & 6.63 & 0.77 & 0.47 & 0.80 & 8.23 \\
\hline$r$ & 0.57 & -0.26 & 0.38 & 0.45 & 0.68 & -0.63 & 0.46 & 0.86 \\
\hline$p$ & $1.26 \times 10^{-16}$ & $1.33 \times 10^{-4}$ & $3.55 \times 10^{-8}$ & $7.48 \times 10^{-5}$ & $6.81 \times 10^{-7}$ & $2.53 \times 10^{-6}$ & $5.58 \times 10^{-4}$ & $5.80 \times 10^{-4}$ \\
\hline
\end{tabular}

${ }^{\mathrm{a}}$ Details of these values are given in section 3 . 
(a)

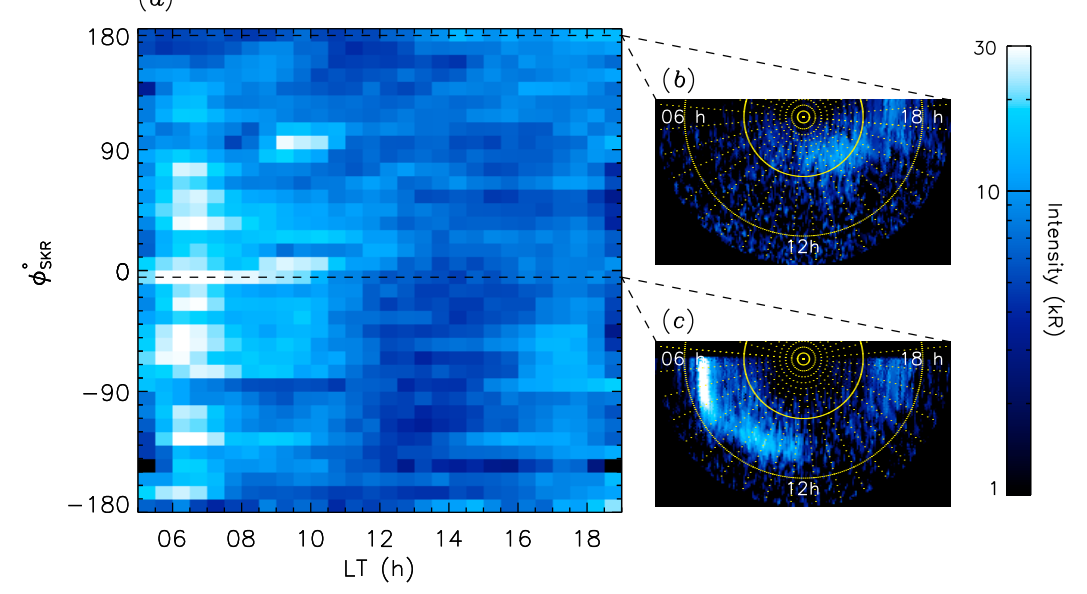

Figure 3. (a) The maximum UV intensity between $7^{\circ}$ and $22^{\circ}$ southern colatitude as functions of LT (in $0.5 \mathrm{~h}$ bins) and SKR phase $\phi_{\mathrm{SKR}}$ (in $10^{\circ}$ bins), along with representative images of the southern oval with phase for (b) $\phi_{\mathrm{SKR}} \simeq 179^{\circ}$ obtained on 9 Feb 2008 and (c) $\phi_{\mathrm{SKR}} \simeq-2^{\circ}$ obtained on 28 Feb 2009 .

\section{North 2009}

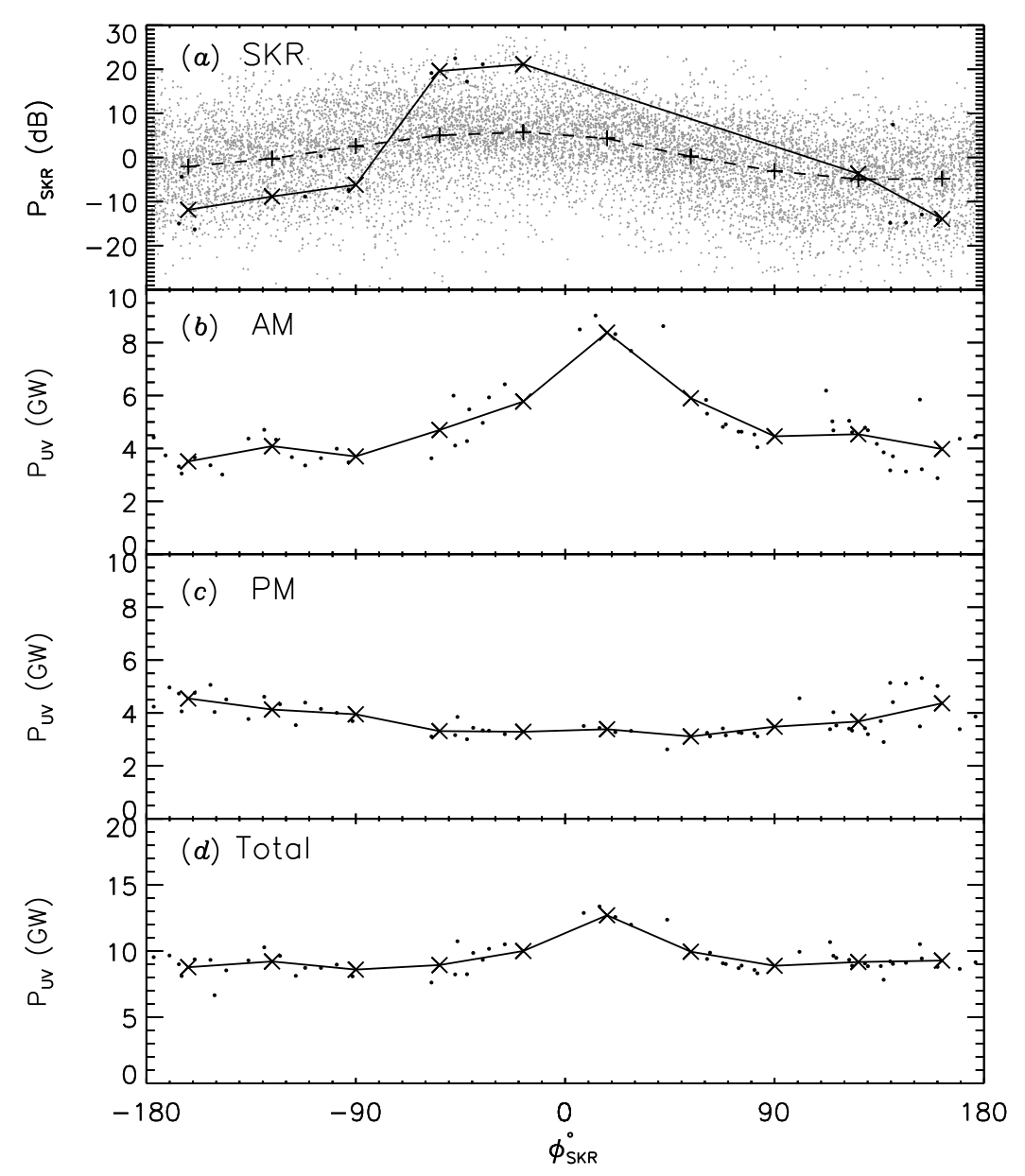

Figure 4. The northern auroral powers obtained in Jan-Mar 2009 versus northern SKR phase in a format similar to Figure 1 except that here the pluses joined by the dashed lines show the mean SKR powers for all the data in ten $36^{\circ}$-wide phase bins and the joined crosses show the mean SKR and HST results in ten $36^{\circ}$-wide phase bins. 
results are also borne out in the statistics of the variations shown in Table 1, which generally exhibit larger overall variation, higher correlation coefficients and lower standard errors than for the south.

\section{Summary}

[9] Despite the physical association of SKR emissions with Saturn's UV auroras made by previous authors, the most significant property of the SKR, i.e., pulsing near the planetary period, has not previously been shown to be present in the auroral data. In this paper we have considered the variation of Saturn's quiet time UV auroral power with SKR phase observed in HST ACS data obtained over the interval 2005-2009. We have shown that for both the north and south the dawnside auroral power exhibits a statistically significant variation by factors of $\sim 3$, with maximum output occurring during peak SKR power, while there is evidence for weaker, opposite behaviour in the duskside power. The total power, being the sum of these two halves, thus varies but not to the same degree as the dawnside on its own. Such behaviour may be indicative of modulation by a rotating current system such as that seen in the magnetometer data, though with larger modulation at dawn than dusk, and which is possibly associated with the $\sim 2^{\circ}$ oscillation in the auroral oval location observed by Nichols et al. [2008]. These results confirm the physical association of the UV aurora and SKR emissions. We note that the SKR power typically oscillates diurnally by orders of magnitude, although the scatter in the SKR power is such that the amplitude averaged over tens of rotations is $\sim 3$ [Kurth et al., 2007, 2008]. Further examination of the northern auroras will be possible as Saturn moves toward northern summer and the view from Earth of the northern pole ameliorates.

[10] Acknowledgments. This work is based on observations made with the NASA/ESA Hubble Space Telescope, obtained at STScI, which is operated by AURA, Inc. for NASA. JDN, SWHC, and AG were supported by STFC grant ST/H002480/1. JTC was supported by NASA grant HSTGO-10862.01-A from STScI to Boston University. JCG and DG were supported by the Belgian Fund for Scientific Research (FNRS) and the PRODEX Programme managed by ESA in collaboration with the Belgian Federal Science Policy Office. LL was supported by the STFC rolling grant to ICL. BC and PZ acknowledge support from the CNES agency. The authors acknowledge the support of ISSI, as this study was discussed by ISSI International Team 178, and thank W. S. Kurth for helpful discussions on the SKR oscillation.

\section{References}

Carbary, J. F., D. G. Mitchell, P. Brandt, E. C. Roelof, and S. M. Krimigis (2008), Periodic tilting of Saturn's plasma sheet, Geophys. Res. Lett., 35, L24101, doi:10.1029/2008GL036339.

Clarke, J. T., et al. (2009), Response of Jupiter's and Saturn's auroral activity to the solar wind, J. Geophys. Res., 114, A05210, doi:10.1029/ 2008JA013694.

Cowley, S. W. H., D. M. Wright, E. J. Bunce, A. C. Carter, M. K. Dougherty, G. Giampieri, J. D. Nichols, and T. R. Robinson (2006), Cassini observa- tions of planetary-period magnetic field oscillations in Saturn's magnetosphere: Doppler shifts and phase motion, Geophys. Res. Lett., 33, L07104, doi:10.1029/2005GL025522.

Galopeau, P. H. M., P. Zarka, and D. Le Quéau (1995), Source location of Saturn's kilometric radiation: The Kelvin-Helmholtz instability hypothesis, J. Geophys. Res., 100(E12), 26,397-26,410.

Gérard, J.-C., et al. (2006), Saturn's auroral morphology and activity during quiet magnetospheric conditions, J. Geophys. Res., 111, A12210, doi: $10.1029 / 2006$ JA011965.

Grodent, D., J.-C. Gérard, S. W. H. Cowley, E. J. Bunce, and J. T. Clarke (2005), Variable morphology of Saturn's southern ultraviolet aurora, J. Geophys. Res., 110, A07215, doi:10.1029/2004JA010983.

Gurnett, D. A., et al. (2004), The Cassini radio and plasma wave investigation, Space Sci. Rev., 114(1-4), 395-463.

Gurnett, D. A., A. Lecacheux, W. S. Kurth, A. M. Persoon, J. B. Groene, L. Lamy, P. Zarka, and J. F. Carbary (2009), Discovery of a north-south asymmetry in Saturn's radio rotation period, Geophys. Res. Lett., 36, L16102, doi:10.1029/2009GL039621.

Kurth, W. S., et al. (2005), An Earth-like correspondence between Saturn's auroral features and radio emission, Nature, 433(7027), 722-725, doi:10.1038/nature03334.

Kurth, W. S., A. Lecacheux, T. F. Averkamp, J. B. Groene, and D. A. Gurnett (2007), A Saturnian longitude system based on a variable kilometric radiation period, Geophys. Res. Lett., 34, L02201, doi:10.1029/ 2006 GL028336.

Kurth, W. S., T. F. Averkamp, D. A. Gurnett, J. B. Groene, and A. Lecacheux (2008), An update to a Saturnian longitude system based on kilometric radio emissions, J. Geophys. Res., 113, A05222, doi:10.1029/ 2007JA012861.

Lamy, L., P. Zarka, B. Cecconi, S. Hess, and R. Prangé (2008), Modeling of Saturn kilometric radiation arcs and equatorial shadow zone, J. Geophys. Res., 113, A10213, doi:10.1029/2008JA013464.

Lamy, L., B. Cecconi, R. Prangé, P. Zarka, J. D. Nichols, and J. T. Clarke (2009), An auroral oval at the footprint of Saturn's kilometric radio sources, colocated with the UV aurorae, J. Geophys. Res., 114, A10212, doi:10.1029/2009JA014401.

Mitchell, D., et al. (2009), Recurrent energization of plasma in the midnight-to-dawn quadrant of Saturn's magnetosphere, and its relationship to auroral UV and radio emissions, Planet. Space Sci., 57, 1732-1742, doi:10.1016/j.pss.2009.04.002.

Nichols, J. D., J. T. Clarke, S. W. H. Cowley, J. Duval, A. J. Farmer, J.-C. Gérard, D. Grodent, and S. Wannawichian (2008), Oscillation of Saturn's southern auroral oval, J. Geophys. Res., 113, A11205, doi: $10.1029 / 2008$ JA013444.

Nichols, J. D., et al. (2009), Saturn's equinoctial auroras, Geophys. Res. Lett., 36, L24102, doi:10.1029/2009GL041491.

Prangé, R., L. Pallier, K. C. Hansen, R. Howard, A. Vourlidas, G. Courtin, and C. Parkinson (2004), An interplanetary shock traced by planetary auroral storms from the Sun to Saturn, Nature, 432(7013), 78-81, doi:10.1038/nature02986

Press, W. H., S. A. Teukelosky, W. T. Vetterling, and B. P. Flannery (2007), Numerical Recipes: The Art of Scientific Computing, 3rd ed., Cambridge Univ. Press, Cambridge, U. K.

Wu, C. S., and L. C. Lee (1979), A theory of the terrestrial kilometric radiation, Astrophys. J., 230(2), 621-626.

B. Cecconi and P. Zarka, LESIA, Observatoire de Paris, Université Paris Diderot, CNRS, 5 Place Jules Janssen, F-92190 Meudon CEDEX, France.

J. T. Clarke, Center for Space Physics, Boston University, 725 Commonwealth Ave., Boston, MA 02215, USA.

S. W. H. Cowley, A. Grocott, and J. D. Nichols, Department of Physics and Astronomy, University of Leicester, Leicester LE1 7RH, UK. (jdn@ ion.le.ac.uk)

J.-C. Gérard and D. Grodent, Laboratoire de Physique Atmosphérique et Planétaire, Université de Liège, Allee du 6 Aout, 17, B-4000 Liège, Belgium.

L. Lamy, Space and Atmospheric Physics Group, Imperial College London, London SW7 2AZ, UK. 